Jaurnal of Enviranmental Science, Computer Science and Engineering \& Technology

An International Peer Review E-3 Journal of Sciences and Technology

\author{
Available online at www.jecet.org
}

Section B: Computer Science

Research Article

\title{
Comparative Analysis of Swift and Go Programming Languages
}

\author{
Elliot, Kizzy Nkem and Ojekudo, Nathaniel Akpofure, \\ Department of Computer Science, Ignatius Ajuru University of Education, Nigeria
}

Received: 28 November 2019; Revised: 09 December 2019; Accepted: 16 December 2019

\begin{abstract}
Programming languages are the way developers communicate with computers. In the past two or three decades, multiple languages have evolved. Today, a common topic for discussion among software engineers is the comparison of these programming languages. Often times, questions or enquiries are made by students or people who wishes to venture into application development on which programming language will be more effective to learn and use for their programming. However, most times, good answers to these enquiries or questions are not easily found. This paper, presents a comparative analysis between two amongst the several programming languages we have. There two programming language compared in this paper are the go programming language and swift programming language. These languages are compared in terms of parameters such as speed, efficiency as per memory, compilation, usage, OS support and paradigm.
\end{abstract}

Keywords: Comparative Analysis, Swift, Go, Programming Languages, Application development

\section{INTRODUCTION}

Computer programming languages are fascinating and interesting area of study. Almost every year new programming languages are being created and as a matter of fact, a thousand different programming languages and even more have been created in the past few years. The question of which language is 
best is one that consumes a lot of time and energy among computer professionals especially programmers. There are strengths and weaknesses in every language, the strengths can be seen as its advantages whereas, the weaknesses are the disadvantages. The FORTRAN programming language for example is good for the analysis of numerical data, but not so good when used in assembling or organizing large programs. In terms of writing a well-structured and readable program, Pascal is one of the programming languages that are very good for it, but on the contrary, it not as versatile as the $\mathrm{C}$ programming language. $\mathrm{C}++$ embodies powerful object-oriented but it is complex and difficult to learn.

Nevertheless, certain programming languages are closer to each other, while others are more distinct. Depending on their similarity or paradigms, programming languages are categorized into various classes or paradigms that we will look briefly at in this article. Each language has their unique set of keywords and a special syntax for organizing program instructions. Several programming languages have been compared by several authors as to know which one is suited for a particular job. Some of them include: C++, C\#, Java, Python, VB.net and so on. This comparison(s) has helped in creating awareness to application developers and enlightening them on the benefits and drawbacks each language can bring to their software solution. Some of these languages have been compared in terms of reusability, consistency, portability, programmer and tool accessibility, readability, performance, familiarity, expressiveness, etc. Also used as comparison parameters were other criteria such as programming effort, run time efficiency, memory consumption, and database connectivity.

\section{RELATED WORKS}

The programming language comparison as implemented by different programmers and these languages includes C, C++, Java, Perl, Python, Rexx, and Tcl was presented ${ }^{[1]}$. The study compared various properties such as run- time, memory constraints, and reliability. The research described by Prechelt also considered language efficiency. It compares efficiency of four languages. Due to the nature of the study, Prechelt did not use a Relative Complexity Metric, which is a representation of a program's metrics, to statistically compare the programming languages. There are additional variations resulting from different programming styles and different frameworks. Prechelt used Relative Complexity Metric, by implementing the algorithms for comparison on a common hardware configuration.

Oguntunde ${ }^{[2]}$ in his study, did a comparative analysis of some programming languages. In his work, he based his analysis on different groups of scientific, non-scientific and object-oriented programming languages which includes Java, $\mathrm{C}++$.

Pascal, Fortran, and Visual Basic. He presented an algorithm for performing combination and permutation to implement the comparison. Two parameters - memory consumption and running time requirement, were tested. From his result, it could be observed that Object-Oriented program has the smallest running time followed by scientific program then Non-scientific. With the Object-Oriented programming languages, JAVA program is faster than $\mathrm{C}++$, although it required more memory allocation than all other programming languages. With the Scientific programming language, PASCAL is faster than FORTRAN but they both have the same memory space requirements. Lastly, in Nonscientific programming language, BASIC has lesser running time compare to COBOL and it also required less memory space allocation compared to COBOL.

Zakaria et. al. ${ }^{[3]}$ compared six different programming languages which includes PHP, C\#, C++ Java, Visual Basic and Python. According to them, their parameters for comparison are "reusability, reliability, portability, availability of compilers and tools, readability, efficiency, familiarity and expressiveness." In the study, several experiments were made and a number of specific criteria were 
considered to compare their selected programming languages. The study revealed that, due to their internal design and structure, each language is best suited for a specific application domain. C\# has shown a great performance in GUI design. Java has demonstrated better suitability for web programming thanks to J2EE as well as desktop applications. C\# and Java are strongly typed languages, which helps writing secure programs. $\mathrm{C}++$ has outperformed all the languages under study in database connectivity and execution time. This shows the great power and widespread use of the $\mathrm{C}++$ language in the software world. PHP is better suited for web development (such as java) and connectivity to databases (such as $\mathrm{C}++$ ). VB is perfect for the rapid development of GUI software and the scheduling of events. Python can be used to build enterprise applications as a feeder language (scripting language) with other static programming languages. According to the researchers, "It can also be used for quick prototyping as; with python, we can achieve less code-to-task ratio."

The strength of the Rosetta Code. It software is a database of solutions in different languages for specific programming activities, providing a large data collection for review was used ${ }^{[4]}$. Their study is based on 7'087 implementation programs that lead to 745 tasks in 8 commonly used languages (procedural: C and Go; object-oriented: C \# and Java; functional: F \# and Haskell; scripting: Python and Ruby). Their study revealed differences regarding some of the most frequently discussed language features conciseness, performance, failure-proneness. According to them, the key to having highly significant statistical results in their study was the use of a large program chrestomathy: Rosetta Code. The repository can be a valuable resource also for future programming language research that corroborates, or otherwise complements, their findings. Besides using Rosetta Code, researchers can also improve it (by correcting any detected errors) and can increase its research value (by maintaining easily accessible up-to-date statistics) ${ }^{[4]}$.

\section{EXPERIMENTAL}

Comparison of Go and Swift Programming Languages: The comparison of both the swift and Go programming languages is carried out using parameters such as speed in terms of their execution time, efficiency as per memory, compilation, usage, operating system support, and paradigm. The above parameters are used to compare these two programming languages - Swift and Go, so as to identify the very best among them. Hence, these parameters are briefly explained below:

Speed (execution time): How fast a programming language runs when executing a program is determined by the speed. In other words, the speed of a programming language has to do with the time it takes programming language to run or execute a line or some lines of codes. This is to say that, the speed of a programming language(s) is a function of time. All programming languages differ in terms of speed, i.e., there execution time. Some programming languages are faster while some are slower. In the experiment conducted by $\operatorname{Marcin}^{[5]}$ after running 30 times loops with both Swift and Go programming showed that in terms of performance as per speed, the Swift program was executed faster with $0 \mathrm{~m} 0.416 \mathrm{~s}$ than that of Go $0 \mathrm{~m} 0.592 \mathrm{~s}$, although the difference is not much.

Efficiency: The efficiency of a programming language has to do with the ability of the programming language to make good use of resources which are available to it to produce the best possible result or outcome. These resources can be in terms of memory, time, etc. but for the purpose of this paper, we are considering efficiency in terms of memory, that is, how well a programming language can manage the memory of the computer in which it is running on while executing a set or some sets of programs. 
Compilation: This has to do with how computer programs written in high level language which is human language are converted to low level language which is the machine language. In terms of compilation, some programming languages are quick to compile than the others.

Operating Systems support: Some programming languages are designed to run on some operating systems, in other words, some OS only support some programming languages.

Hence, a programming language supported by an operating system may not be supported by another operating system.

Paradigm: A programming language paradigm has to do with the style or a way of programming in that language. The paradigm could be structured, procedural, object-oriented, functional or logical etc.

Therefore, table 1 below shows comparison of the Swift programming language and Go programming language in terms of the above defined parameters - Speed, Efficiency, Compilation, OS Support, and Paradigm.

Table 1: Comparative analysis of the Swift and Go programming languages

\begin{tabular}{|l|l|l|}
\hline Basis of Comparison & Swift & Go \\
\hline Speed (execution time) & Very fast & Fast \\
\hline Efficiency (as per memory) & Efficient & Efficient \\
\hline Compilation & $\begin{array}{l}\text { Not so quick to compile } \\
\text { compared to Go. }\end{array}$ & $\begin{array}{l}\text { Compiles quickly compared to } \\
\text { Swift. }\end{array}$ \\
\hline Operating system support & Used for iOS & Used for Windows and Linux \\
\hline Paradigm & $\begin{array}{l}\text { It is an object-oriented, } \\
\text { imperative, and functional } \\
\text { programming language. }\end{array}$ & $\begin{array}{l}\text { It is a procedural, functional and } \\
\text { concurrent programming } \\
\text { language. }\end{array}$ \\
\hline
\end{tabular}

\section{RESULTS AND DISCUSSION}

Result from the above comparison shows that in terms of speed, both programming languages are fast but the Swift language is faster than that of Go. In terms of efficiency, both languages are efficient when it comes to memory usage. When it comes to compilation, swift is quick to compile compared to the Go programming language? While the Swift is an object-oriented, imperative, and functional programming language which runs on iOS; the Go is a procedural, functional and concurrent programming language which runs on Linux and Windows operating systems.

Evolution of Programming Languages: Programming languages are the way developers communicate instructions to computers - just like we use natural languages to communicate with one another. In both cases multiple languages have evolved. With the 1960s came the quest for the perfect programming language. In a landmark article, there had already been 700 programming languages he speculated on what the next 700 would be like. After that, there were thousands of programming languages that emerged. Through academia, programming languages are seen to function like an ecosystem: new languages emerge and old languages are substituted if they do not develop. Nevertheless, compared to natural languages, the programming language environment shifts at a much higher rate ${ }^{[6]}$.

Programming language evolution as an important subject which affects developers, language designers and researchers and argues that there is need for older programming languages to be improved upon 
otherwise they will be displaced by newer ones. There are two main forces influencing programming language evolution and they include: external and internal. External forces include hardware transition, industry trends and advances in science. For example, as a way to facilitate coding on multicore architectures, most typically object-oriented programming languages such as Java, C \#and C++ have introduced functional programming features. For example, Java's latest version (Java 8) supports lambda expressions and a new declarative array storage API ${ }^{[7]}$.

On the other hand, internal forces come from clumsiness or inefficiencies in existing versions of the language and may not necessarily be influenced by the ecosystem outside of the language. For example, language designers may need to fix bugs or design mistakes. In addition, developers may become frustrated with the verbosity of certain code patterns and practices. For example, iterating over a container before Java 5 required substantial set-up work. The for-each construct introduced in Java 5 addressed that specific issue. Therefore, programming languages that do not respond to these two powers are assumed to be automatically substituted. Some of the programming languages that were popular are: Ada, Algol, Cobol, Pascal, Delphi, and Snobol. Evolving programming languages is however challenging at various levels. According to Joseph ${ }^{[8]}$, "every change is an incompatible change. A risk/benefit analysis is always required."

Firstly, it is important to note that evaluating a proposed language change is difficult; language designers often lack the infrastructure to assess the change. Most often, they rely on mailing lists and surveys from the community. Mainstream programming language programmers have recently argued for the use of real-world data to push issues related to language evolution ${ }^{[9]}$. Nevertheless, it can be challenging to carry out tests because they take time. As a result, evaluations are rarely performed, which can contribute to subjective judgments. Older and infrequently used features for example may still be put in future language versions in order to retain backward compatibility and at the same time increase the language's complexity (e.g., FORTRAN 77 to Fortran 90). Similarly, developers have few means to understand the compatibility of their codebase with a new language version.

Secondly, there may be a negative impact on the language developers. For example, if two language versions are incompatible, the new language version may be rejected by developers. Older versions, though, are often not supported by language developers. Developers may therefore miss significant bug fixes and security updates. It's a big industrial problem. Python 2 and 3, for instance, are largely incompatible. This topic has an effect on big companies like Google who have millions of lines of code written in Python 2. Every change to a programming language has the potential to affect programs written in that language. A recent study shows that developers lack awareness of the subsequent possible incompatibilities ${ }^{[10]}$.

Programming Language Syntax and Semantics: Virtually all programming languages have some basic software definition building blocks or processes and transformations that are added to them. Such primitives are described by syntactic and semantic rules that explain their respective structure and context.

A language syntax defines potential symbol combinations that shape a code that is syntactically correct. Semantics (whether formal or hard-coded in a reference implementation) manages the sense assigned to a mixture of symbols. This paper discusses textual syntax since most languages are textual in nature. As stated by Oguntunde ${ }^{[2]}$, "the static semantics defines restrictions on the structure of valid texts which are difficult or impossible to express in standard syntactic formalisms. Static semiconducting requires basically certain semiconducting laws that can be tested at compile time for compiled languages. These include ensuring that each identifier is declared before use or that the marks on a case statement's arms are distinct." 
Language Processors: Computers in general do not understand high-level languages. A translator is therefore needed to convert the code into its machine language counterpart in order to make computers understand the codes. These translators are called processors of the language. A language processor's work is basically to check the syntactic and semantic correctness of a program's statements. Language processors are of three classes namely: Assembler, Interpreter and compiler.

The Assembler translates codes written in assembly language into object codes that the machine understands; the Interpreter translates a high-level language one statement at a time and carries out the execution of the statement before proceeding to the next statement; while the Compiler translates a program written in high-level language into its machine language equivalent.

Some Programming Paradigms: Programming is a rich discipline and there are quite complicated practical programming languages that one can learn. Luckily, the basic idea behind every programming language are quite simple. To solve a programming problem, the right values need to be chosen. That's why many paradigms should be followed by programming languages. Peter ${ }^{[11]}$, defines a programming paradigm as "an approach to programming a computer based on a mathematical theory or a coherent set of principles. Put simply, a programming paradigm is a style or a way of programming." Every paradigm follows a collection of ideas that make it the best for a problem of some kind. Some paradigms are best suited to problems with a wide number of related information abstractions arranged in a hierarchy, several others are suited to converting and manipulating complicated mathematical constructs according to functional laws, while others are best suited to responsive problems, i.e. problems consisting of responses to sequences of external events. To date, Java, Prolog, and Esterel are respectively notable languages that support these three paradigms.

Jing $^{[12]}$ classified programming paradigms into imperative and declarative paradigms and stated that there are other paradigms that are generally considered subtypes of the two aforementioned ones. He placed the structured, procedural as well as the object-oriented paradigms as sub-paradigms under the Imperative paradigm and also the functional and logic paradigms as sub-paradigms under the Declarative paradigm. In addition to the two basic paradigms mentioned above, Jing also argued that there are several other types of paradigms such as concurrent paradigm, generic, paradigm, and so on.

However, Milena and Dusan ${ }^{[13]}$ recognises in their paper four programming paradigms which they argued are the four main programming paradigms in Computer Science. According to them, these paradigms include: Imperative Paradigm, Object-Oriented Paradigm, Functional Paradigm, and Logic Paradigm. Hence, the summary of some of the subtypes of the imperative and declarative paradigms listed above are explained below:

i. Procedural programming: This approach stresses the basic computer design process. Among formal and imperative method, there is no distinction. It has the potential to recycle the software and because of its reusability, it was boon at the period when it was in operation. Examples of programming languages under this programming paradigm include: C, C++, Java, ColdFusion, Pascal etc.

ii. Object oriented programming: Here, codes are written as a set of classes and objects designed for interaction. The smallest and most simple element is the object and all sorts of computation are done only on the objects. Further emphasis is placed on information rather than process. It can handle almost all kind of real-life problems which are today in scenario. Some advantages of the OOP paradigm are: data security, inheritance, code reusability as well as flexibility and abstraction. Examples of programming languages 
under this programming paradigm include: Simula, Java, $\mathrm{C}++$, Objective-C, Visual Basic .NET, Python, Ruby etc.

iii. Functional programming: The functional programming paradigms are grounded in mathematics and are free of grammar. This paradigm's key principle is the implementation of sequence of computational functions. The basic model of abstraction is the method intended for some particular computation and not the structure of the data. Data is loosely linked to functions. They hide their implementation from the functions. Task can be replaced by its principles without modifying the program's purpose. This paradigm is mostly used by some languages like Perl, JavaScript. Many functional programming style examples include: Lisp, Haskwell, Scala, etc.

iv. Logic programming: This framework can be called the abstract programming model and it is used to solve logical issues such as puzzles, series, etc. We have a knowledge base in logic programming that we learn before and that produces results along with the problem and knowledge base that is presented to the computer. This definition of knowledge base is not accessible in ordinary programming languages, but while using the framework of artificial intelligence, machine learning we have some systems such as the system of vision which uses the same process. The main emphasis in logical programming is on knowledge base and the problem. Program execution is much like logical assertion argument, e.g., prolog.

Swift Programming Language: Swift is a new programming language created by Apple ${ }^{[14]}$ in 2014 . According to Apple ${ }^{[14]}$ "it allows for developing applications for the new version of operating systems of Apple: iOS and OS X. The Shift programming language was designed to be an eventual replacement for Objective-C." Apple's goal is to deliver a safer, simpler, more versatile, faster, funnier and more programmable programming language than Objective-C to promote the development of applications for Apple platforms ${ }^{[15]}$. Swift is a great way to build applications, whether you're running programming for devices, desktops, servers, or anything else. It is also a quick, safe, and engaging programming language. The language combines the good sides of modern languages with expertise from Apple's broader culture and the vast contributions of its open-source community.

Swift is user-friendly especially to new programmers. It's an industrial-quality programming language that's as expressive and enjoyable as a scripting language. Writing codes in a swift playground allows you to experiment with program and immediately see the results, without building and running an app overhead. Quoting Singh and Kaur ${ }^{[16]}$, "the programming language defines away large classes of common programming errors by adopting modern programming patterns, for example, variables are always initialized before use, array indices are checked for out-of-bounds errors, integers are checked for overflow, and memory is managed automatically."

Swift as a programming language just like the Objective-C is both object-oriented and imperative. The only thing that differentiates it from Objective-C is that it embodies functional programming. Closures, charts and filters are some examples of this. The swift code is compiled and configured to make the most of modern hardware. The design of both the syntax and standard library was based on a guiding principle which has it that the best performance should also be the easiest way to write your code. Its combination of safety and speed make Swift an excellent choice for everything from "Hello, world!" to an entire operating system ${ }^{[17]}$. The language blends powerful inference form and pattern matching with a traditional, lightweight syntax, allowing for clear and precise expression of complex ideas. Code is therefore not only easier to compose, but also easier to read and comprehend. Based on these facts, a 
study on Swift is needed to ascertain if it can be a programming language that can cope with the modern times and whether it can promote the development of applications for Apple platforms.

Go Programming Language: GO is an object-oriented programming language with a C-like syntax which run on Linux and Windows operating systems. The language was developed by Rob Pike, Ken Thompson and others at Google in 2007. The language was designed and developed to meet certain problems experienced by software engineers at Google and was later made public and open source in November 2009. This company typically used Java, C++ and Python for their large projects and some of their projects they claimed to be indeed very large which makes some problems with the development cycle more evident. It supports a mixture of static and dynamic typing, and is designed to be safe and efficient. A primary motivation is its compilation speed ${ }^{[18]}$.

Many languages, programming platforms, operating systems and their developers are what influences the GO programming language. Go embraces multi-paradigm such as procedural, concurrent and functional. Traditionally, its syntax comes from C, but it has made a lot of changes to improve features such as simplicity and security. Rather than classes, Go has objects and structs. It has no concept of inheritance. Frank et al. ${ }^{[19]}$ states in their work that "There are no clear subtype statements in Go, but structural subtypes are implied in some instances."

Thompson and Pike's experience and involvement in the development of Unix, C, Plan 9, Inferno and Limbo clearly influenced the design of GO. GO's inter-process communication is largely influenced by CSP and its successors. The language makes use of typed channels and these channels may be buffered and unbuffered; the process synchronization structure defined by Hoare and implemented in Newsqueak is executed by its "SELECT" statement. The compiler suite used by GO is based on Plan 9's compiler suite. In terms of modularity and declarations, GO is influenced by Modula, Pascal and Oberon programming languages ${ }^{[20]}$.

\section{CONCLUSION}

Conclusively, both Swift and Go have their own set of advantages, based on the willingness of the programmer to grasp the necessity of the real world. Swift is regarded as a bottom-up language from top to bottom. It is best suited for composing secured codes. It offers powerful generics when compared to Go to enable the writing of general-purpose libraries to extend the language's power. Go is very easy to learn. In terms of developer efficiency, Go vs Swift takes different paths. Swift checks for a wide range of type-level features common to $\mathrm{C} \#$, Java, and $\mathrm{C}++$ programmers. With the clarification of the above capabilities and options for both Go and Swift languages, programmers are at liberty to choose a language which conforms with their project specifications and requirements. It all boils down to the nature of the project, its specific requirement, and the ease with a language of the developer at the end of the day.

\section{REFERENCES}

1. L. Prechelt, An Empirical Comparison of C, C++, Java, Perl, Python, Rexx, and Tcl for a Search/String-Processing Program. Technical Report, 2005.

2. O. Oguntunde, Comparative Analysis of Some Programming languages. Transnational Journal of Science And Technology, 2012, 2 (5), 107 - 118. Retrieved on the 28th of October, 2019 from: http://www.tjournal.org/tjst_june_2012/8.pdf?i=1

3. A. Zakaria, E. Oualid, S. Kaushik., and Chitrang Pandit, Comparative Studies of Six Programming Languages. 2015, Retrieved on the 27th October, 2019 from: 
https://www.researchgate.net/publication/274572185_Comparative_Studies_of_Six_Program ming_Languages.

4. N. Sebastian, and A. Carlo, A Comparative Study of Programming Languages in Rosetta Code. IEEE/ACM 37th IEEE International Conference on Software Engineering. 2015, Retrieved on the 29th of October, 2019 from: https://www.researchgate. net/publication/ 265252296_A_Comparative_Study_of_Programming_Languages_in_Rosetta_Code

5. K. Marcin, Swift vs Go vs Python battle, 2015, Retrieved on the 28th of October, 2019 from: http://www.marcinkliks.pl/2015/02/22/swift-vs-others/

6. V. Peter, Programming Paradigms for Dummies: What Every Programmer Should Know, 2014, Retrieved on the 28th of October, 2019 from: https://www.info.ucl .ac.be/ pvr/ VanRoy Chapter. pdf

7. U. Raoul-Gabriel, Programming Language Evolution. 2017, Retrieved on the 27th October, 2019 from: https://www.cl.cam.ac.uk/techreports/UCAM-CL-TR-902.pdf

8. D. Joseph, Kinds of compatibility: Source, binary, and behavioural, 2008.

9. G. Briant, Language designer's notebook: Quantitative language design. 2010

10. D. Jens, J. Kamil, and B. Premek, What Java developers know about compatibility, and why these matters. Empirical Software Engineering, 2015, 1.

11. J. Peter, The next 700 programming languages. Communications of the ACM, 1996, 9 (3), 157 $-166$.

12. C. Jing, A Brief Survey of Programming Paradigms. Retrieved on the 28th of October, 2019from:https://medium.com/@jingchenjc2019/a-brief-survey-of-programming-paradigms $207543 \mathrm{a} 84 \mathrm{e} 2 \mathrm{~b}$

13. V. Milena, and T. Dusan, The Role of Programming Paradigms in the First Programming Courses. 2008, Retrieved on the 29th of October, 2019 from: http://elib.mi.sanu.ac.rs/files/journals/tm/21/tm1122.pdf

14. Apple, 2015. Swift Programming Language. Retrieved on the 29th of October, 2019 from: https://developer.apple.com/swift/

15. B. Cory. Apple's Swift Programming Language. Retrieved on the 29th of October, 2019 from:https://www.techrepublic.com/article/apples-swift-programming-language-the-smartpersons-guide/

16. B. Singh, and R. Kaur, Raising Performance of iPhone using Swift Language over Other Programming Languages. International Journal of Advance Research, Ideas and Innovations in Technology, 2017, 3 (6), 991 - 994.

17. G. Cristian, P. Jordán, B. Cristina, G. Bustelo, and M. Juan, Swift vs. Objective-C: A New Programming Language. International Journal of Artificial Intelligence and Interactive Multimedia, 2015, 3(3), $74-81$.

18. R. Pike, Go, The Programming Language. 2009, Retrieved on the 28th of October, 2019 from: http://golang.org/doc/talks/go_talk-20091030.pdf

19. S. Frank, C. Nicholas, and N. James, Evaluating the GO Programming Language with Design Patterns. International Journal of Advance Research, Ideas and Innovations in Technology, 
2010, 4 (2), 144 - 153. Retrieved on the 29th of October, 2019 from: https://ncameron.org/papers/schmager plateau10.pdf

20. N. Wirth, and J. Gutknecht, Project Oberon: The Design of an Operating System and Compiler. ACM Press/Addison-Wesley Publishing Co., New York, NY, USA. 1992.

\section{Corresponding author: Ojekudo, Nathaniel Akpofure,}

Department of Computer Science, Ignatius Ajuru University of Education, Nigeria nathojekudo@gmail.com 